\title{
Low-dimensional dynamics of a turbulent axisymmetric wake
}

\author{
G. Rigas $\dagger$, A. R. Oxlade, A. S. Morgans, J. F. Morrison \\ Department of Aeronautics, Imperial College London, London SW7 2AZ, UK \\ (Received ?; revised ?; accepted ?. - To be entered by editorial office)
}

The coherent structures of a turbulent wake generated behind a bluff three-dimensional axisymmetric body are investigated experimentally at a diameter based Reynolds number $\sim 2 \times 10^{5}$. Proper orthogonal decomposition of base pressure measurements indicates that the most energetic coherent structures retain the structure of the symmetry-breaking laminar instabilities and manifest as unsteady vortex shedding with azimuthal wavenumber $m= \pm 1$. In a rotating reference frame, the shedding preserves the reflectional symmetry and is linked with a reflectionally symmetric mean pressure distribution on the base. Due to a slow rotation of symmetry plane of the turbulent wake around the axis of the body, statistical axisymmetry is recovered in the time average. The ratio of the timescales associated with the slow rotation of the symmetry plane and the vortex shedding is of order 100 .

Key words:

\section{Introduction}

Flows behind bluff bodies are of fundamental importance to many industries, in particular the transport industry, where the aerodynamic drag arising from such flows can be the dominant source of vehicle fuel-burn and $\mathrm{CO}_{2}$ emissions (Hucho 1998). At low Reynolds numbers, corresponding to laminar and transitional flows, hydrodynamic stability theory has aided understanding of the dynamics of bluff-body wakes (Meliga et al. 2009). However, flows pertinent to the transport industry involve high Reynolds numbers and turbulent wakes. Despite their turbulence, such wake flows exhibit organisation which manifests as coherent flow structures: these are usually associated with increased noise, structural fatigue and drag. Understanding the underlying wake dynamics in the turbulent regime is of paramount importance for the development of practical control strategies.

In this paper, we analyse the dynamics of the coherent structures of the turbulent wake of a bluff three-dimensional axisymmetric body with a blunt trailing edge. We show, perhaps surprisingly given that $R e_{D} \sim 2 \times 10^{5}$, that these dynamics can in fact be linked to the hydrodynamic instabilities observed during the transitional regimes at low Reynolds numbers. Here, $R e_{D}=U_{\infty} D / \nu$ is the Reynolds number, $U_{\infty}$ is the free-stream velocity, $D$ is the diameter of the body and $\nu$ is the fluid kinematic viscosity. An important feature of our study is the use of only body-mounted sensing. In real transport flows, full flow-field measurements are impractical, with sensors embedded in/attached to the body being the only feasible option. In the context of drag reduction, only those structures which influence the pressure force on the base of the bluff-body are of importance. We

$\dagger$ Email address for correspondence: g.rigas@imperial.ac.uk 
therefore conduct our analysis entirely using pressure measurements on the base of the body.

The paper first presents an overview of laminar (\$1.1) and turbulent ( $\$ 1.2)$ axisymmetric wake behaviour, before presenting the experimental setup ( $(2)$, results ( 33$)$ and conclusions $(\S 4)$.

\subsection{Laminar/transitional regime}

It has been generally acknowledged that the flow behind an axisymmetric body is dominated by a global instability with azimuthal wavenumber $|m|=1$, which plays an important role during the transition of the laminar wake. The flow past blunt-based axisymmetric bluff bodies has been considered in various direct numerical simulations (Sanmiguel-Rojas et al. 2009; Bohorquez et al. 2011; Bury \& Jardin 2012). These studies showed that the wake undergoes two successive bifurcations at low Reynolds numbers from an axisymmetric steady state. The first is a steady bifurcation with $|m|=1$ which leads to a double-threaded wake structure; although the flow is still steady, it is no longer axisymmetric but preserves reflectional symmetry about a fixed plane that passes along the axis of the body. For larger Reynolds numbers, a Hopf bifurcation with $m= \pm 1$ leads to unsteady flow characterised by the shedding of streamwise-oriented, alternating hairpin-like vortices; the flow preserves the reflectional symmetry due to the eccentricity of the vortex shedding. The threshold of the bifurcations depends on the length-todiameter ratio of the body, $L / D$; for a body with $L / D=7$, these regimes were observed at Reynolds numbers close to 450 and 590 (Bury \& Jardin 2012).

Further departure from the second critical Reynolds number renders the wake chaotic. During the chaotic regime the reflectional symmetry is broken; however, phases of reflectional symmetry whose flow topology resembles that of the reflectional symmetric state are randomly interrupted by dramatic changes of the azimuthal position of the symmetry plane (Bury \& Jardin 2012). The reflectional symmetry plane exists in an instantaneous sense, but it is randomly and intermittently re-oriented on a very long time scale.

Beyond the second critical Reynolds number, Bury \& Jardin (2012) identified a second mode with a frequency lower than the natural shedding frequency and which dominates the drag fluctuations. Bohorquez et al. (2011) also reported a second peak appearing in the spectra of the axial velocity component at a frequency approximately one quarter of the shedding.

Similar regimes are observed in the wake behind a sphere (Pier 2008; Tomboulides \& Orszag 2000) and a disk (Natarajan \& Acrivos 1993) suggesting that the transitional behaviour of axisymmetric wakes follows a similar scenario during the initial stages at low Reynolds numbers of $\mathcal{O}(100)$.

\subsection{Turbulent regime}

Symmetry considerations are central not only to the study of transitional phenomena, as described above, but also to fully developed turbulence. Although bifurcations break the symmetries on the way to turbulence, fully developed turbulence is known to restore (all or some) of the possible symmetries in a statistical sense, at very high Reynolds numbers (Frisch 1996).

At high Reynolds numbers, the bifurcating oscillatory state observed in the laminar wakes manifests as shedding of large-scale structures. Early experiments in the turbulent wake behind axisymmetric bodies, such as disks and spheres, (Achenbach 1974; Taneda 1978; Fuchs et al. 1979) showed that the predominant flow structures in the near wake are coherent antisymmetric modes with $|m|=1$, which are shed at a constant frequency. However, and in contrast with the laminar regime, all of the above studies of turbu- 

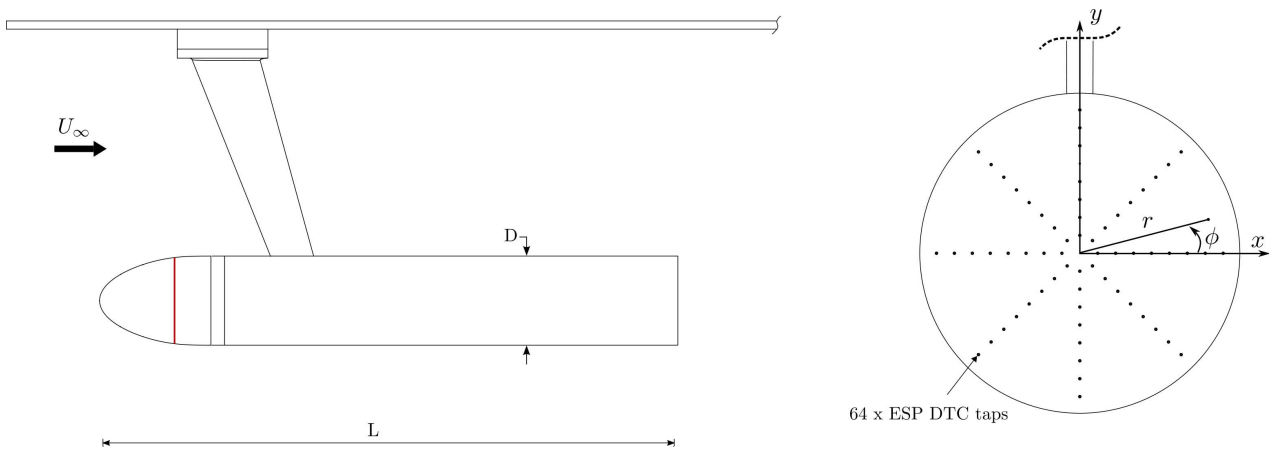

FiguRE 1. Schematic of axisymmetric bluff-body (a) Side view, (b) Rear view.

lent wakes showed that the separation point rotates randomly around the body above Reynolds number 1000, approximately. In more recent experiments behind blunt-based axisymmetric bodies, Sevilla \& Martínez-Bazán (2004) and Grandemange et al. (2012) measured a dominant antisymmetric mode with $|m|=1$ at a Strouhal frequency of $S t \approx 0.2$.

\section{Experimental setup}

Experiments were conducted in a closed circuit wind tunnel, the working section of which measures $0.91 \mathrm{~m} \times 0.91 \mathrm{~m} \times 4.8 \mathrm{~m}$. The contraction ratio is $9: 1$ and the free-stream turbulence intensity is less than $0.1 \%$. A PID controller with a set-point variation of less than $0.2 \%$ is used to maintain the free-stream velocity, $U_{\infty}=15 \mathrm{~ms}^{-1}$.

The wake is generated by an axisymmetric bluff-body with blunt trailing edge, a schematic of which is shown in figure 1 . The body diameter, $D$, is $196.5 \mathrm{~mm}$ and the length-to-diameter ratio, $L / D$, is 6.48 . The nose employs a modified super ellipse profile (Lin et al. 1992) with an aspect ratio of 2.5. The boundary layer is conditioned by a $2 \mathrm{~mm}$ wide strip of 120 grit emery paper located at $z / L=-0.884$ (approximately the point of minimum pressure) followed by a $25 \mathrm{~mm}$ wide strip at $z / L=-0.784$. The axisymmetric body is supported in the centre of the wind tunnel test section using a NACA 0015/0030 blended aerofoil, such that a constant thickness of $11 \mathrm{~mm}$ was maintained throughout (Qubain 2009). More details for the model are given by Oxlade (2013). The Reynolds number based on diameter and boundary layer momentum thickness at separation is $R e_{D}=1.88 \times 10^{5}$ and $R e_{\theta}=2050$ respectively, corresponding to turbulent wake and a turbulent boundary layer separation.

The base of the body is instrumented with 64 static pressure taps equi-spaced on a uniform polar grid with $\delta r=0.056 D$ and $\delta \phi=45^{\circ}$ in the radial and azimuthal direction, respectively (figure 1b). Static pressure is measured using an ESP-64HD DTC pressure scanner and a Chell CANdaq 14 bit D/A converter with a sampling frequency of $225 \mathrm{~Hz}$. A total of 19,200 s of data was acquired over sixteen independent experiments, providing approximately 2000 independent measurements with a $95 \%$ uncertainty of approximately $0.45 \%$ and $1 \%$ in time average and rms pressure respectively. Pressure measurements are expressed as a pressure coefficient, non-dimensionalised by the free-stream dynamic head $1 / 2 \rho U_{\infty}^{2}$. Frequency spectra are calculated using blocks of $2^{14}$ data points and a Hanning window with $50 \%$ overlap. Frequencies are expressed as Strouhal numbers, $S t=f D / U_{\infty}$. 


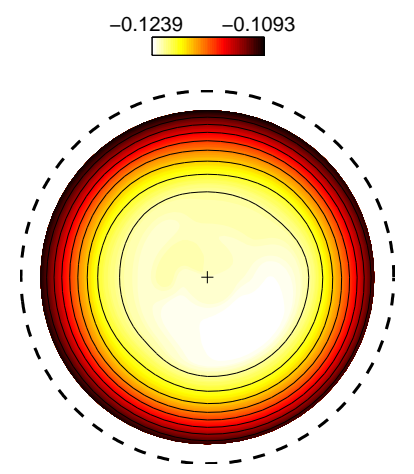

(a)

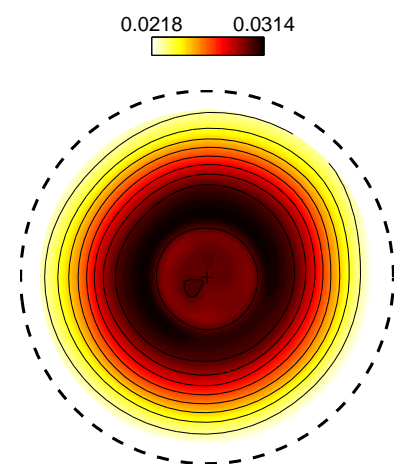

(b)

Figure 2. Pressure distribution on the base of the body: (a) mean and (b) root-mean-square of the fluctuating component. The dashed circle indicates the body diameter.

\section{Results}

\subsection{Base pressure distribution}

The mean and root-mean-square pressure distribution on the base of the body are shown in figure 2. A region of constant low pressure extends from the body axis up to approximately $r / D=0.2$, with pressure recovery increasing towards the edge of the body. Conversely, the fluctuating pressure exhibits a maximum at approximately $r / D=0.15$. Both pressure distributions are axisymmetric to within $\pm 1 \%$.

\subsection{Azimuthal modes}

A Fourier decomposition of the pressure signal $p(r, \phi, t)$ in the azimuthal direction gives azimuthal modes,

$$
p_{m}(r, t)=\frac{1}{2 \pi} \int_{0}^{2 \pi} p(r, \phi, t) e^{-i m \phi} \mathrm{d} \phi .
$$

Coherent structures are identified in figure 3 calculating the azimuthal spectral energy $\bar{\Phi}_{m}(S t)$ distributed over $S t$, and averaged over the radius, such that

$$
\overline{p_{m}^{2}}=\frac{8}{D^{2}} \int_{0}^{D / 2} \int_{0}^{\infty} S t \Phi_{m}(r, S t) \mathrm{d}(\log S t) r \mathrm{~d} r .
$$

The dominant mode shapes and their associated frequencies are shown in table 1.

Spectral peaks at $S t \approx 0.2(d)$ are associated with the global oscillatory mode of the wake: vortex shedding with azimuthal wavenumber $m= \pm 1$. The frequency and shape are consistent with previous experimental observations from bodies of similar geometry (Sevilla \& Martínez-Bazán 2004; Grandemange et al. 2012). The $m= \pm 1$ spectra show also peaks at $S t \approx 0.1(c)$, which are close to the subharmonic of the shedding mode. The same frequency is also observed in the modes $m= \pm 2$.

We also observe that the $m= \pm 1$ mode oscillates with a very low frequency (VLF) centred at $S t \approx 0.002(a)$, which is approximately two orders of magnitude less than the shedding frequency. A similar timescale, $t \sim 10^{3} \mathrm{D} / U_{\infty}$, has been reported recently in the three dimensional wake of an Ahmed body for high Reynolds numbers (Grandemange et al. 2013). For the Ahmed body, this long timescale was linked to the random shifts of the recirculation region between two preferred reflectional-symmetry-breaking positions leading to a statistically symmetric wake. The dynamics of this structure for the axisymmetric wake are investigated in $\S 3.4$. 


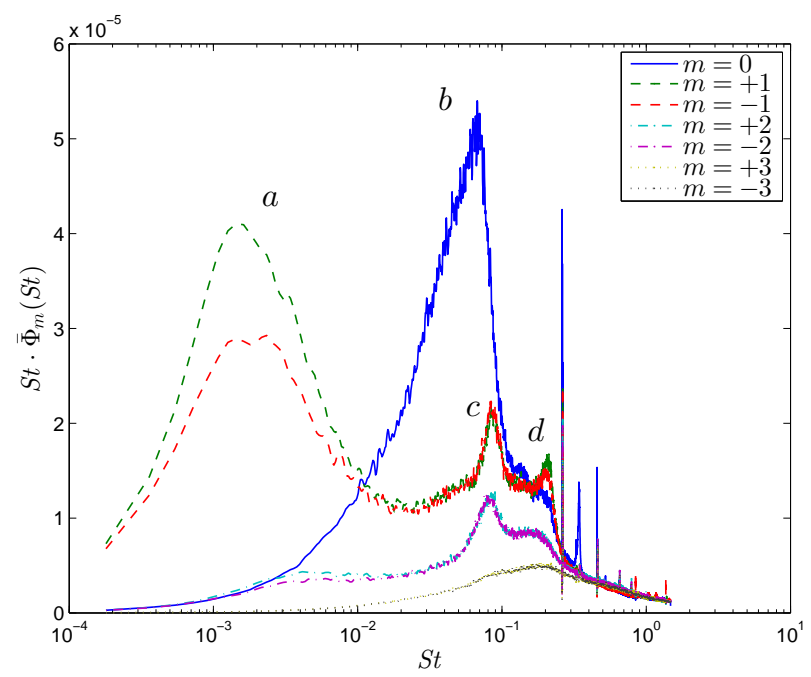

FIGURE 3. Premultiplied pressure spectra of the azimuthal modes on the base of the axisymmetric body. Spectra are radially averaged and the areas underneath correspond to energies; spectral peaks show where the energy is concentrated.

$\begin{array}{lccccc} & a & b & c & d & \text { POD Modes } \\ m=0 & & 0.06 & & & 3 \\ m= \pm 1 & 0.002 & & 0.1 & 0.2 & 1,2 \\ m= \pm 2 & & & 0.1 & & 4,5\end{array}$

TABle 1. Dominant modes and associated Strouhal numbers based on energy content.

An axisymmetric $(m=0)$ pulsation of the vortex cores, known as "bubble pumping" (Berger et al. 1990), is identified at a low frequency of $S t=0.06(b)$. The intensity of the axisymmetric pulsation increases as the axis of the body is approached. The frequency and shape of this mode is very close to the low frequency oscillation of the wake observed by Bohorquez et al. (2011) and Bury \& Jardin (2012) for a body of similar geometry in the transitional regime at low Reynolds numbers.

\subsection{POD decomposition}

Proper orthogonal decomposition has been shown to be an effective way to systematically extract coherent structures of turbulent flows based on their energy content (Lumley 1970; Holmes et al. 2012). Figure 4 shows the energy distribution of the 20 most energetic POD modes of the base pressure. It can be seen that the first three modes carry $\sim 72 \%$ of the total energy.

Figure 5 shows the first five POD modes of the base pressure and the corresponding premultiplied spectral density $\Phi$ of the POD coefficients. The coefficients are computed by projecting each mode onto the instantaneous pressure field (Holmes et al. 2012). The first five POD modes capture all the coherent structures, based on the presence of spectral peaks in figure 3; no peaks are detected in the spectra of the remaining POD amplitudes.

The first two most energetic POD modes (modes 1 and 2) correspond to the modes with azimuthal wavenumber $m= \pm 1$ in table 1 , the third POD mode to the axisymmetric $m=0$ pulsation, and the fourth and fifth to $m= \pm 2$ modes. 


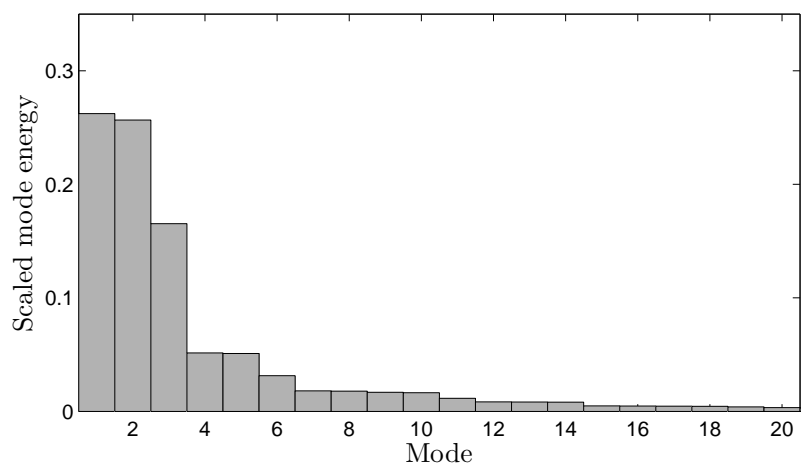

Figure 4. Energy of the first 20 POD modes.
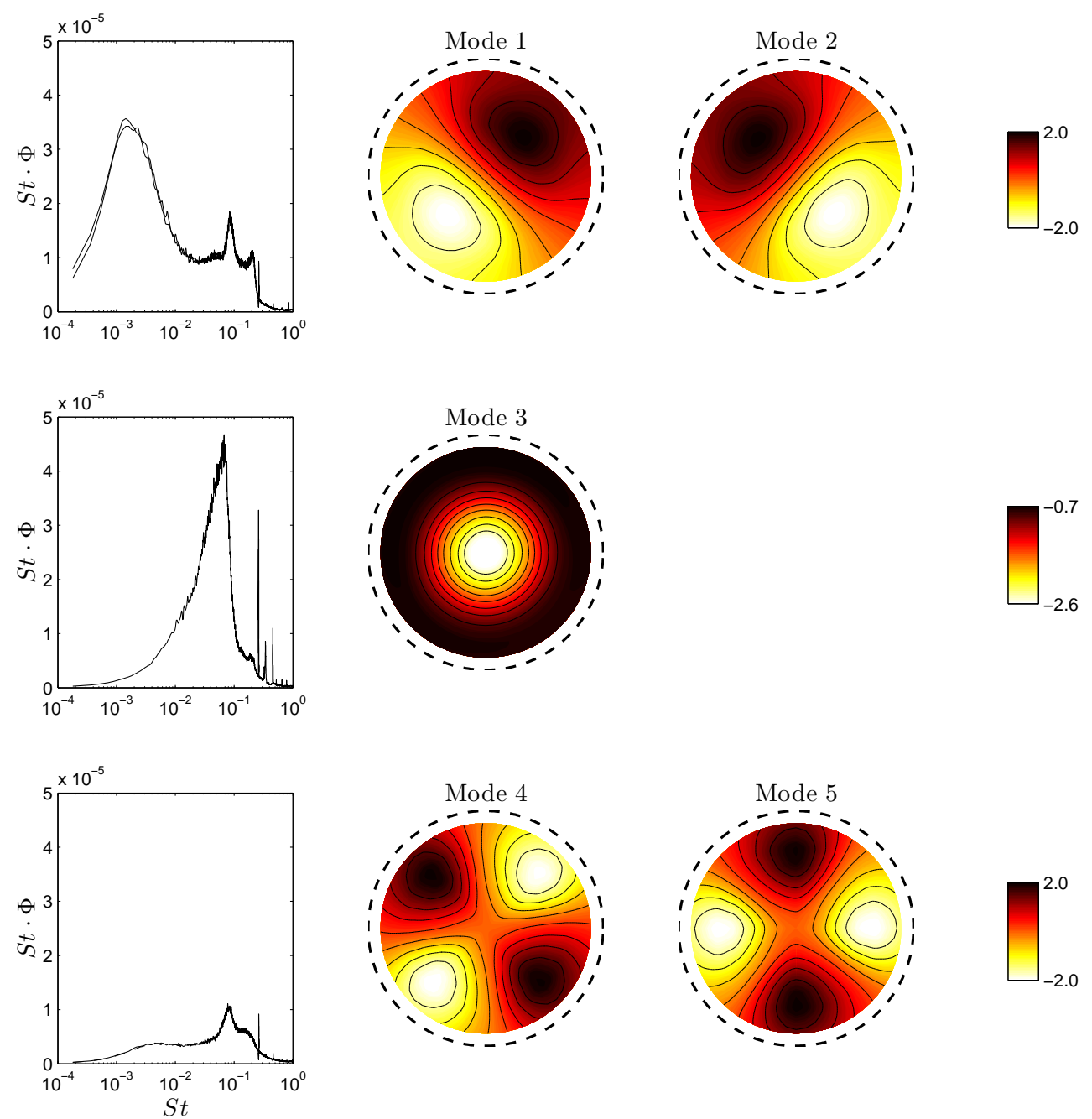

FIgURE 5. First five POD modes and premultiplied spectra of their amplitudes: $(\mathrm{a}-\mathrm{c})$ antisymmetric modes 1-2 with $|m|=1$; (d-e) axisymmetric mode 3 with $m=0$ and, (f-h) antisymmetric modes $4-5$ with $|m|=2$. 


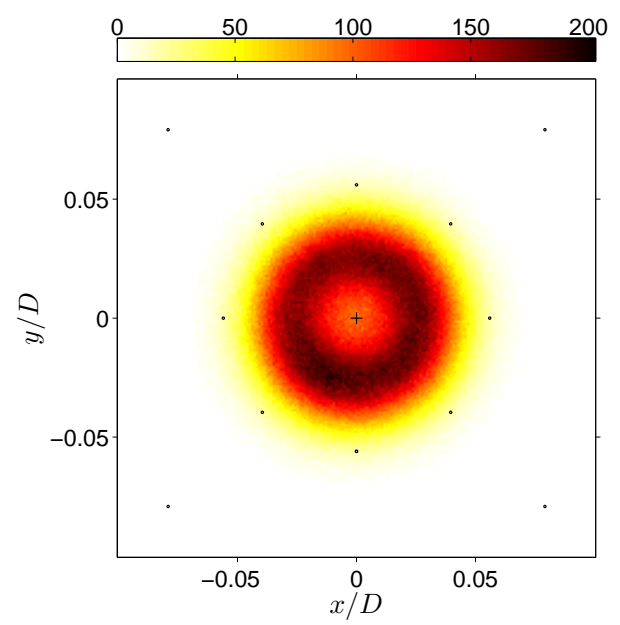

(a)

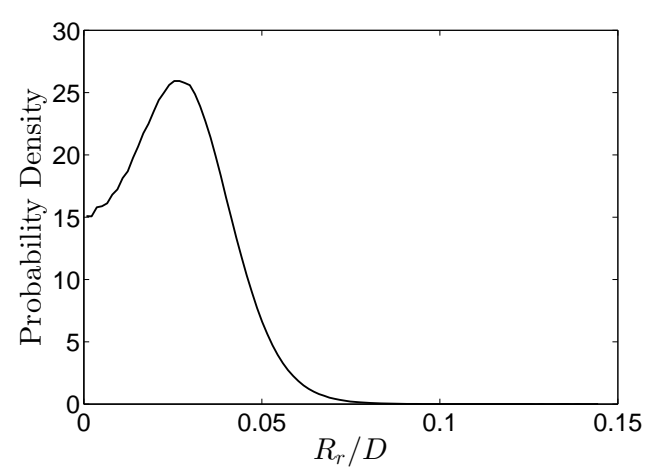

(b)

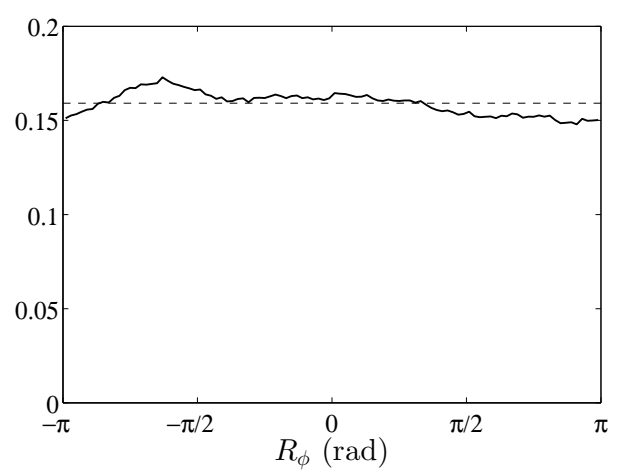

(c)

Figure 6. Probability density of Center-of-Pressure position: (a) two-dimensional, (b-c) one dimensional; radial and azimuthal respectively. Dashed line: the uniform distribution.

\subsection{On the symmetry of the flow}

Insight to the symmetry of the wake and its link to the coherent structures of the turbulent wake is given by the Centre-of-Pressure $(\mathrm{CoP})$, calculated from the space-averaged pressure, defined on the Cartesian coordinate system of the base, $\mathbf{x}=(x, y)$, as

$$
\mathbf{R}(t)=\frac{1}{\int p(t) d A} \int_{A} p(t) \mathbf{x} d A
$$

where $A$ is the area of the base of the body. The CoP provides a direct way to quantify the magnitude of the asymmetry in the turbulent regime: a zero value (CoP lies on the centre) will correspond to an $\mathcal{R}_{\pi}$-symmetric flow $(\phi \rightarrow-\phi ; P \rightarrow P$ ), whereas departure from this value will correspond to an increased asymmetry of the flow.

The two-dimensional probability density function of the $\mathrm{CoP}$ is plotted in figure 6 , together with the one-dimensional components in the radial $\left(R_{r}\right)$ and azimuthal $\left(R_{\phi}\right)$ directions. It shows that the most probable location of the CoP lies on a circle with $R_{r}=0.03 D$ centered on the base of the body, indicating a tendency to lock to this value. The probability of the azimuthal position of the $\mathrm{CoP}$ is consistent with a uniform distribution within the experimental error (small deviations from the uniform distribution 


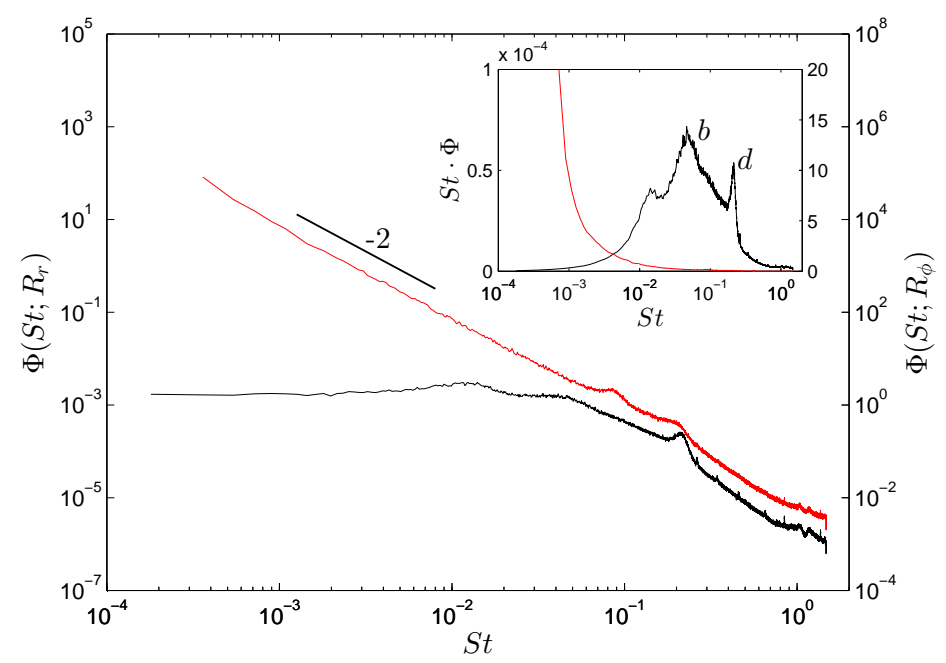

FiguRE 7. Spectral density of Centre-of-Pressure in polar coordinates: - , radial $R_{r} ;-$, azimuthal $R_{\phi}$. The inset shows premultiplied spectra.

are mainly due to imperfect alignment of the experimental setup, which is also evident in the energy imbalance of the VLF mode in figures 3 and 4). Due to the uniform distribution, an axisymmetric pressure field is obtained in the long time average. However, the non-zero mean radial value of the $\mathrm{CoP}$ provides strong evidence that the wake is asymmetric, for a fixed angle of the CoP, on average.

Further information on the dynamic behaviour of the coherent structures is provided by examining the spectra of the $\mathrm{CoP}$, plotted in figure 7 . In the spectrum of the angular component, a power-law with an exponent close to -2 is obtained at very low frequencies (VLF). This is analogous to the results of Grandemange et al. (2013), and consistent with Brownian dynamics (Brown \& Ahlers 2006). Hence the VLF oscillation is a random rotation of the $\mathrm{CoP}$ in the azimuthal direction around the axis of the body. The spectrum of the radial component exhibits a clear peak $(d)$ at the vortex shedding mode, $S t \approx 0.2$. Below that frequency, a power-law roll-off is observed, with the radial spectral density saturating at low frequencies. This gradient change in the spectral density plotted on logarithmic scale, manifests as peak (b) in the premultiplied spectrum and coincides with the energy associated with the bubble pumping.

In order to identify the asymmetry of the flow, the mean pressure statistics were calculated in the rotating reference frame of the CoP by rotating the data based on the instantaneous angle of the CoP (Grandemange 2013). In the rotating frame, the axisymmetry of the mean and root-mean-square pressure observed in the long-time average is lost, as shown in figure 8 . Both distributions are characterised by reflectional symmetry and an azimuthal modulation of $m=1$. The $\mathcal{R}_{\pi}$ symmetry of the wake is broken: calculation of the radial distance of the CoP from the centre of the body confirms the value of $R_{r}=0.03 D$ found above for the mean pressure distribution.

The averaging procedure of the base pressure distribution on the rotating reference frame reveals a flow topology that resembles that of the reflectionally symmetric topology observed in the laminar wake, immediately after the second symmetry-breaking bifurcation. In this laminar regime, vortices are shed periodically off-centre of the axis creating a reflectionally symmetric distribution; the angle of the symmetry plane is constant and is determined from the initial conditions. However, for the turbulent regime, the low frequency rotation of the symmetry plane results in an axisymmetric pressure distribution 


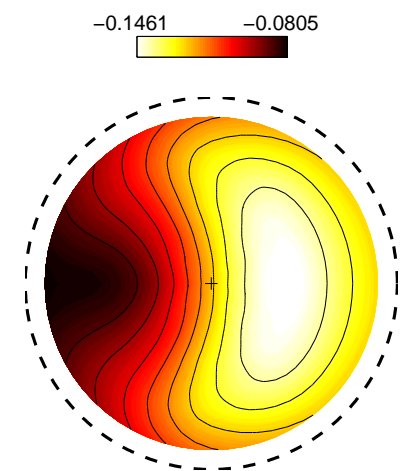

(a)

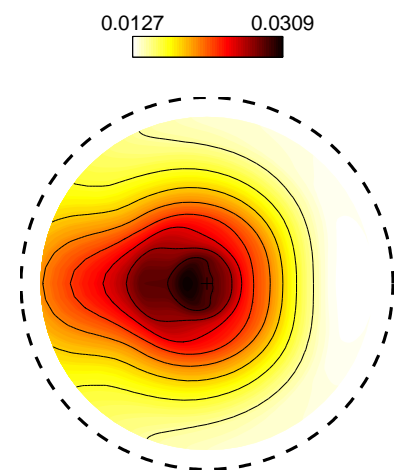

(b)

Figure 8. Pressure distribution on the base of the body on the rotating reference frame of the Center-of-Pressure: (a): Mean and (b) root-mean-square.

on the base, see figure 2, due to the uniform variation in the orientation of the vortex shedding.

Therefore, the turbulent state explores a continuum of metastable symmetry-breaking patterns $\left(R_{r} \neq 0\right)$, the angle of which is arbitrary. However, the mean value of the twodimensional probability density lies on the centre of the body $\left(R_{r}=0\right)$, and the flow recovers $\mathcal{R}_{\pi}$ symmetry, provided the averaging is performed over sufficiently long time.

\section{Conclusions}

In the present investigation, the coherent structures that describe the large-scale dynamics of a turbulent three-dimensional wake have been characterised in detail from pressure measurements performed on the base of an axisymmetric bluff body.

The symmetry-breaking instabilities of the transitional wake observed at $R e_{D}<1000$ (Bohorquez et al. 2011; Bury \& Jardin 2012) before the chaotic regime, persist at high Reynolds numbers (here $R e_{D} \sim 2 \times 10^{5}$ ). Although the wake is turbulent, the large-scale coherent structures associated with it retain the structure of the laminar instabilities in a statistical sense. These are large-scale anti-symmetric oscillations with $m= \pm 1$ at a frequency of $S t_{D} \approx 0.2$, known as vortex shedding, reminiscent of the unsteady bifurcated state observed in laminar flows.

The shedding is asymmetric and it is linked with an asymmetric mean pressure distribution on the base with $m=1$, which both rotate randomly around the axis of the body at a frequency of $S t_{D} \approx 0.002$. These distributions preserve the reflectional symmetry in the rotating reference frame. Due to this slow rotation the turbulent wake recovers axisymmetry in the long time average.

The dynamics of these two processes impose two well-separated timescales for the evolution of the flow: a short timescale, $t \sim 5 D / U_{\infty}$, associated with the periodic shedding of vortices and a long one, $t \sim 5 \times 10^{2} D / U_{\infty}$, due to the variation of the shedding angle. These two timescales are in agreement with the ones found in the turbulent wake of a rectilinear three-dimensional body (Grandemange et al. 2013) suggesting universal slow dynamics associated to symmetry-breaking modes of three dimensional turbulent wakes. In contrast with the rectilinear-body wake, which has two possible symmetry-breaking states (bistable wake), the axisymmetric-body wake possesses an infinite number of states depending on the azimuthal angle of shedding (multistable wake).

These results provide further insight to the dynamics of three dimensional turbulent 
wakes which can be used for the design of model-based control strategies.

This work was supported by the Engineering and Physical Sciences Research Council (grant number EP/I005684).

\section{REFERENCES}

Achenbach, E. 1974 Vortex shedding from spheres. J. Fluid Mech. 62, 209-221.

Berger, E., Scholz, D. \& Schumm, M. 1990 Coherent vortex structures in the wake of a sphere and a circular disk at rest and under forced vibrations. J. Fluids Struct. 4, 231-257.

Bohorquez, P., Sanmiguel-Rojas, E., Sevilla, A., Jiménez-González, J. I. \& MartínezBAZÁn, C. 2011 Stability and dynamics of the laminar wake past a slender blunt-based axisymmetric body. J. Fluid Mech. 676, 110-144.

Brown, E. \& Ahlers, G. 2006 Rotations and cessations of the large-scale circulation in turbulent Rayleigh-Bénard convection. J. Fluid Mech. 568, 351-386.

Bury, Y. \& JARDin, T. 2012 Transitions to chaos in the wake of an axisymmetric bluff body. Phys. Lett. A 376, 3219-3222.

Frisch, U. 1996 Turbulence. Cambridge University Press, Cambridge, UK .

Fuchs, H. V., Mercker, E. \& Michel, U. 1979 Large-scale coherent structures in the wake of axisymmetric bodies. J. Fluid Mech. 93, 185-207.

Grandemange, M. 2013 Analysis and control of three-dimensional turbulent wakes: from axisymmetric bodies to real road vehicles. PhD thesis, ENSTA ParisTech.

Grandemange, M., Gohlke, M. \& Cadot, O. 2013 Turbulent wake past a three-dimensional blunt body. Part 1. Global modes and bi-stability. J. Fluid Mech. 722, 51-84.

Grandemange, M., Gohlke, M., Parezanović, V. \& Cadot, O. 2012 On experimental sensitivity analysis of the turbulent wake from an axisymmetric blunt trailing edge. Phys. Fluids 24, 035106.

Holmes, P., Lumley, J. L., Berkooz, G. \& Rowley, C. W. 2012 Turbulence, Coherent Structures, Dynamical systems and Symmetry; 2nd ed.. Cambridge University Press.

Hucho, W.-H., ed. 1998 Aerodynamics of road vehicles, 4th edn. Society of Automotive Engineers, SAE.

Lin, N., ReED, H. L. \& SARIC, W. S. 1992 Effect of leading-edge geometry on boundary-layer receptivity to freestream sound. In Instability, Transition, and Turbulence, pp. 421-440. Springer.

Lumley, J. L. 1970 Stochastic tools in turbulence. Academic Press, New York.

Meliga, P., Chomaz, J.-M. \& Sipp, D. 2009 Global mode interaction and pattern selection in the wake of a disk: a weakly nonlinear expansion. J. Fluid Mech. 633, 159-189.

Natarajan, R. \& Acrivos, A. 1993 The instability of the steady flow past spheres and disks. J. Fluid Mech. 254, 323-344.

OxLADE, A. R. 2013 High-frequency periodic jet forcing of a turbulent axisymmetric wake. PhD thesis, Imperial College.

PIER, B. 2008 Local and global instabilities in the wake of a sphere. J. Fluid Mech. 603, 39-62.

Qubain, A. 2009 Active control of a turbulent bluff body wake. PhD thesis, Imperial College.

Sanmiguel-Rojas, E., Sevilla, A., Martínez-Bazán, C. \& Chomaz, J.-M. 2009 Global mode analysis of axisymmetric bluff-body wakes: Stabilization by base bleed. Phys. Fluids 21, 114102 .

Sevilla, A. \& Martínez-BazÁn, C. 2004 Vortex shedding in high Reynolds number axisymmetric bluff-body wakes: Local linear instability and global bleed control. Phys. Fluids 16, 3460-3469.

TANEDA, S. 1978 Visual observations of the flow past a sphere at Reynolds numbers between $10^{4}$ and $10^{6}$. J. Fluid Mech. 85, 187-192.

Tomboulides, A. G. \& Orszag, S. A. 2000 Numerical investigation of transitional and weak turbulent flow past a sphere. J. Fluid Mech. 416, 45-73. 